\title{
GESTIÓN DE LA TECNOLOGÍA, INNOVACIÓN Y COMPETITIVIDAD EMPRESARIAL
}

Por Juan Arturo Ortega Gómez *

* Ingeniero Electricista

Master of Science, instituto Metalúrgico de Zhadov, Marilpol-Ucrania

Especialista en Gestión de Tecnología, Universidad de los Andes, Bogotấ.

Especialista en Gestión de Producción, Calidad y Tecnología, Universidad Politécnica de Madrid.

Profesor de la Unidad de Gestión Tecnológica del Departamento de Producción e investigación de operaciones de la facultad de Ingeniería de la Universidad delvalle.

\section{RESUMEN}

La actual crisis que atraviesa la economía colombiana, y que alcanza a los países miembros de la Comunidad Andina de Naciones - C.A.N. - ha puesto en dificultades al sector productivo, especialmente a las Pequeñas y Medianas Empresas - PYMES -, que ven en peligro su supervivencia, sin vislumbrar alternativas para mantener a sus clientes, o en el mejor de los casos abrir nuevos mercados. En este artículo se presenta una nueva visión de la gestión empresarial basada en la gestión de la tecnología 
y la capacidad de innovación como una estrategia para incrementar la competitividad del negocio.

\section{ABSTRACT}

The crisis that the colombian economy is presently going through and which is extensive to the country members of the Andean Nations Community (Comunidad Andina de Naciones C.A.N.) has decreased the survival chance of many Small and Medium Enterprises -SMES without alternatives for opening new markets. In this article, a new vision of management of Technology and Innovations is presented as an strategy for increasing business competitivity.

\section{INTRODUCCIÓN}

La actual crisis económica que atraviesa la sociedad colombiana y que afecta a la mayoría de los paises de la Comunidad Andina de Naciones, ha generado un decrecimiento en la actividad comercial hasta márgenes insostenibles para un grupo importante de empresas que ven en pelizro su supervivencia sin vislumbrar estrategias que permitan su sostenimiento en el mercado, y en el mejor de los casos, la diversificación y apertura de nuevos clientes.

De otra parte, cada vez se reconoce con mayor claridad en el contexto de la estrategia empresarial la importancia de la innovación tecnológica de productos y procesos para sobrevivir a las presiones competitivas del mercado. Sin embargo la capacidad tecnológica que permite la innovación es el resultado de un proceso que demanda de la gerencia de la empresa una orientación estratégica para la identificación y selección de negocios, inversiones en tecnologías y un esfuerzo sostenido de formación y desarrollo de los recursos humanos.

Las empresas con vocación innovadora siempre están orientadas a crear nuevos clientes, a abrir nuevos mercados, aprovechando sistemáticamente su capacidad tecnológica acumulada de aprender haciendo durante varios años e identificando tendencias y oportunidades del mercado: acuerdos comerciales binacionales y multinacionales.

Entonces decir actualmente que las empresas necesitan fortalecer su capacidad tecnológica para ser más innovadoras y competitivas en las nuevas condiciones de mercados abiertos, estamos de acuerdo que no se considera ya ningún descubrimiento del fenómeno sociotecno-económico en que vivimos, pero para aquellas empresas que lo sean desde ahora conscientes y realmente deseen ganar participación en los mercados, será el comienzo del camino hacia una verdadera estrategia competitiva: la estrategia tecnológica.

En el presente artículo se propone un modelo conceptual que muestra claramente la relación entre la función de Gestión de la Tecnología en la empresa, la formulación de estrategias y la cartera de proyectos tecnológicos para la generación de ventajas competitivas.

\section{LA GESTIÓN DE LA TECNOLOGÍA EN LAS EMPRESAS}

"Herramienta gerencial para desarrollar ventajas competitivas"

Es el momento tal vez más adecuado para definir 
la gestión de la tecnología como el conjunto de funciones y responsabilidades de la dirección general relacionadas con adquisición, transferencia y generación de tecnologías requeridas por la organización para la innovación y el mejoramiento de procesos y productos orientados a oportunidades y necesidades del mercado.

Como resultado de la gestión de tecnología la empresa deberá definir una estrategia tecnológica que esté orientada con la estrategia empresarial y la apoye en los siguientes aspectos: innovación en productos, servicios y mercados, organización de los sistemas de producción e inversión en tecnologias que le permitan creary mantener ventajas competitivas en el mercado.

\section{La Gestión de Tecnología y la innovación Empresarial}
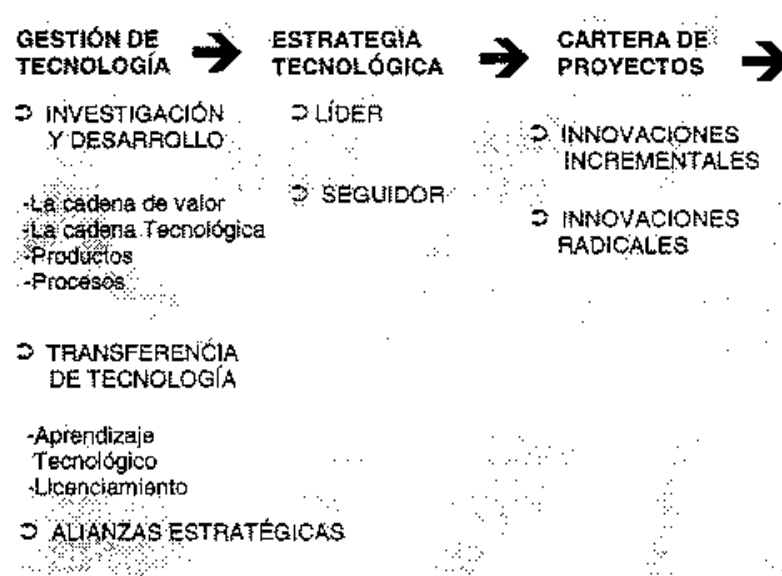

- ESPECIALIZACIÓN

Existe una clara relación entre tecnología, estrategia, innovación y ventaja competitiva, pero las elecciones tecnológicas y la gestión de la innovación en la empresa no es un proceso natural, todo lo contrario reúne características tan complejas que combinan por una parte decisiones intuitivas espontáneas, propias de procesos de cambio para aprovechar oportunidades del entorno (nuevos productos y mercados), y de otra parte actividades sistematizadas (mercadeo, diseño, producción, otras) como reflejo de políticas y cultura arraigadas en la organización que caracterizan su comportamiento tecnológico.

De gran interés resulta el análisis del comportamiento y el desempeño empresarial en la gestión de la tecnología, principalmente en las pequeñas y medianas empresas, para deducir estrategias exitosas hacia la innovación.

De acuerdo con conclusiones de estudios empíricos realizados en lberoamérica(1) y en Colombia(2) sobre la importancia de la innovación en pequeñas y medianas empresas (con menos de 199 empleados) pertenecientes a diferentes subsectores industriales, dejan ver las principales motivaciones y dificultades para adelantar proyectos innovadores.

VENTANA

COMPETITIVA

2 LDERAZCO

EN COSTOS

2 LIDERAZCO EN

DIFERENCIACION

- Las ideas innovadoras surgen desde dentro de la empresa, orientadas por las necesidades y oportunidades del mercado.

- Hay una correlación significativa entre esfuerzo tecnológico, ventas, exportaciones, productividad y utilidades.

Entre empresas tradicionales (no innovadoras) como mayores dificultades encontradas podemos señalar:

- Deficiencias en el análisis de tendencias del mercado y en la planeación y formulación de estrategias. 
- Actitud reactiva ante las amenazas y las oportunidades del mercado.

- El esfuerzo se reduce o se centra en el desarrollo técnico, prestando poca atención a los análisis y oportunidades del mercado.

\section{LA ESTRATEGIA EMPRESARIAL Y TECNOLOGICA}

La estrategia empresarial según los planteamientos de $M$. Porter( 3 ) consiste en la formulación y ejecución de una serie integrada de decisiones basadas en las necesidades del cliente, las cuales incluyen la identificación de una posición definida en el mercado: respecto a ofrecer productos o servicios a bajo costo 0 diferenciados de mayor valor, segmentos, zonas geográficas, integraciones y alianzas estratégicas y decisiones sobre inversiones tecnológicas que le permitan a la empresa convertirse en líder o seguidor en el mercado.

La estrategia empresarial puede construirse de un proceso formalizado de planeación, como resultado, por ejemplo, de estudios de tendencias del mercado o ejercicios de planeación estratégica realizados con la asesoría de una firma consultora contratada por empresas generalmente grandes. En particular en la pequeña y mediana empresa, en donde con frecuencia el propietario es también el gerente, la orientación de la estrategia es el resultado de decisiones muy influenciadas por el estilo personal del gerente.

La empresa debe orientar la estrategia a partir del propósito claro de alcanzar una posición en el mercado con base en las capacidades tecnológicas acumuladas. En este sentido, la estrategia es la fuerza mediadora entre la organización y su entorno, es decir entre las dimensiones interna y externa de la empresa. El objetivo es encontrar una posición favorable en el mercado que se corresponda con las capacidades y recursos de la empresa y que sea sostenible.

La estrategia tecnológica debe ser considerada como un elemento de la estrategia empresarial slobaly, por tanto, debe ser consistente con las decisiones y actuaciones en las diferentes áreas funcionales de la empresa. Principalmente la estrategia tecnológica deberá apoyar la estrategia empresarial en el ámbito externo para la apertura y conservación de mercados; por medio de la innovación de productos y servicios, y en el ámbito interno para la innovación y el mejoramiento continuo de los procesos de la empresa.

Las innovaciones más exitosas, como resultado de la ejecución de la estrategia tecnológica, serán aquellas que consideren la interacción entre la capacidad tecnológica de la empresa y las necesidades del mercado.

Teniendo en cuenta las anteriores consideraciones al momento de decidiry desarrollar una estrategia tecnológica adecuada, la empresa necesita conocer con claridad sus capacidades tecnológicas internas y aquellas áreas que necesita desarrollar.

Una vez que hemos ubicado a la estrategia tecnológica como un elemento de la estrategia competitiva de la empresa, el paso siguiente consiste en estructurar la cartera de proyectos tecnológicos. En este sentido un aspecto importante es estudiar los mecanismos de acceso a las fuentes de tecnología. 
Si consideramos ahora la participación de especialistas de diferentes áreas del conocimiento: física, química, biotecnología, etc., el nivel tecnológico de pruebas y ensayos, y los importantes recursos de inversión en equipos e instrumentación que caracterizan los proyectos de innovaciones tecnológicas en productos y procesos productivos, excluyendo a las multinacionales, nos queda claro que será muy difícil para las empresas, por no decir imposible, particularmente para las pequeñas y medianas, acometer proyectos de innovación tecnológica sin contar con sevicios externos para adelantar experimentos tecnológicos que se necesiten y que pueden ser realizados en algunas áreas del conocimiento en centros de investigación de universidades y otras fuentes externas, muchas veces en condiciones técnicas y económicas más favorables que realizando toda la fase de investigación y desarrollo solamente con los recursos propios de la empresa.

No se trata de contar en la nómina de la empresa con todos los premios Nobel de física o química, ni tener los laboratorios de investigación más sofisticados y costosos, porque difícilmente una evaluación financiera lo justificaría, la solución es, por una parte, desarrollar la capacidad de gestión de la organización para la formación permanente de su capital humano en aquellas áreas estratégicas que se requiere mejorar, y de otra parte, demostrar habilidad para acceder a fuentes externas de información y conocimiento: clientes, consultorías tecnológicas, centros de investización y desarrollo, universidades, ferias y congresos especializados, entre otros.

La primera parte de la solución se inicia formando a los directivos de la empresa en gerencia o zestión de la tecnología, en programas de especialización como el ofrecido por la Universidad del Valle o en cursos para gerentes promovidos en el Servicio Nacional de Aprendizaje - SENA - en el marco de los programas de competitividad y desarrollo tecnológico para el sector productivo. La segunda parte, podemos concluir que es resultado de la primera.

Es importante aclarar que la capacidad tecnológica se adquiere por la empresa, no solamente como un proceso natural, resultado de la función de producción y el uso rutinario de la tecnología incorporada en equipos, sino especialmente como una estrategia deliberada: y consciente de la gerencia de la empresa que se propone implantar la gestión de tecnología que le permita desarrollar o adquirir diversas capacidades tecnológicas aplicadas a la innovación de productos y servicios. El fortalecimiento de estas capacidadés debería ser el punto de partida para cualquier esfuerzo innovador.

\section{Bibliografía}

1. CYTED-D, Estudio "Cien empresas innovadoras en lberoamérica, Santiago de Chile, CEPAL, 1992.

2. ACOLTEC, Informe del Programa de Gestión de la Imnovación Tecnológica, Santafé de Bogotá, ACOLTEC, 1998.

3. PORTER, Michel, The competitive advantage of nations, New York, Free Press, 1990. 